\title{
Implementation and Application of NFC Based on Android
}

\author{
Aobo Zhang ${ }^{1, a}$
}

${ }^{1}$ Department of Computer Science, North China Electric Power University, Baoding 071000, China;

a1002411381@qq.com

Keywords: NFC, Android

\begin{abstract}
Near Field Communication (NFC) is a simple and contactless communication technology, and it's very popular in consuming electronic filed. Android is one of the most popular mobile operating system, and it's combined with NFC which can greatly promote the development of both sides. We mainly pay attention to the NFC implementation framework of on Android, and describes in detail the realization of key technology, and the practical test results is given.
\end{abstract}

\section{Introduction}

Near Field Communication (NFC) is a set of short-range wireless technologies, typically requiring a distance of $4 \mathrm{~cm}$ or less to initiate a connection. NFC allows you to share small payloads of data between an NFC tag and an Android-powered device, or between two Android-powered devices.

There are three kinds of NFC terminal working mode:

1) Active mode: As a card reader, NFC terminal take the initiative to make their rf (radio frequency) field to recognize and read or write the NFC device.

2) Passive mode: NFC terminal can be modeled as a smart card to read or write, it only responses passively in the rf field from other devices.

3) Two-way mode: Both sides make a rf to establish a point-to-point communication.

\section{Frame and implementation of the Android NFC}

In order to support NFC function, Android allows applications to read in the label, and interact in NDEF (NFC Data Exchange Format) message format. Tags can also be another device, namely the NFC device works in simulation mode.

The Android NFC software architecture defines the following data structures:

In the NFC Android framework implementation, it follows Android universal Service basic structure model and the Manager. NFC Manager provides application programming interfaces that communicate via Binder and Service as shown in Figure.

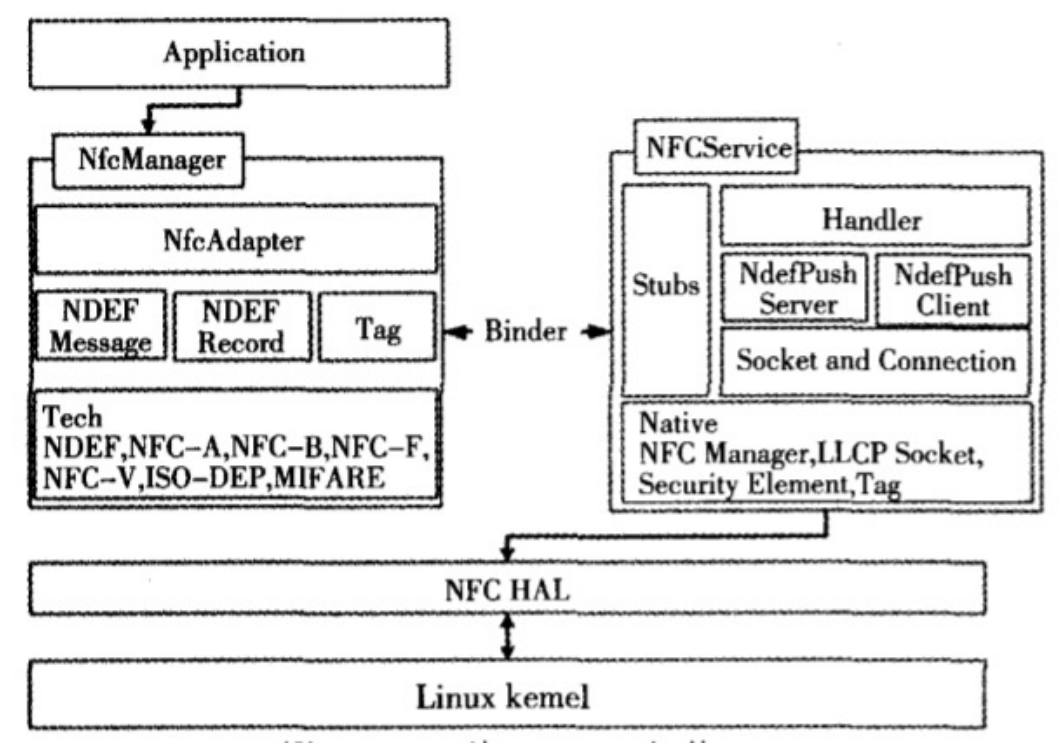




\section{Identification tags}

In the NFC about reading/writing tags, labels simulation and point-to-point communications applications, we describes the implementation of Android focusing on the most commonly used identification tags. Identify the basic processes of a label as shown in Figure.

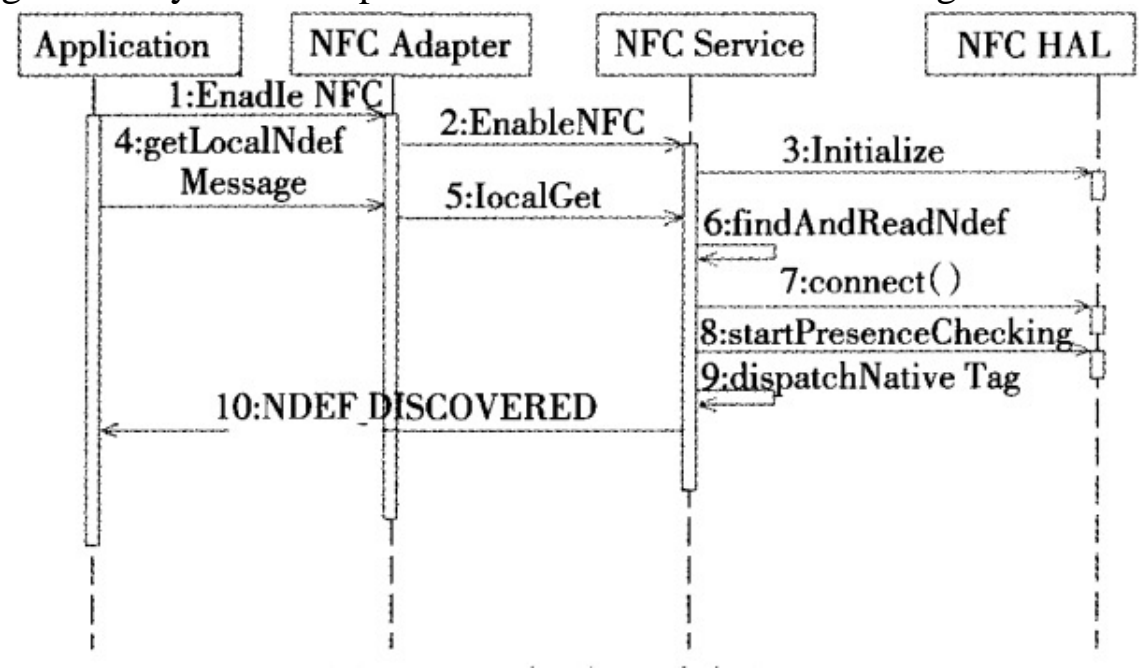

\section{4. the Thread of Android NFC}

After opening NFC in system settings feature, NFC hardware devices can be detected within the effective distance range label. After the data has been read, which Intent messages sent to the system, the application receives the message, parse the tag data and processing is performed.

1) Receive the Intent messaging program segment.

2) Analytic NDEF messages program.

3) As data displayed, running the test result is shown in Figure.

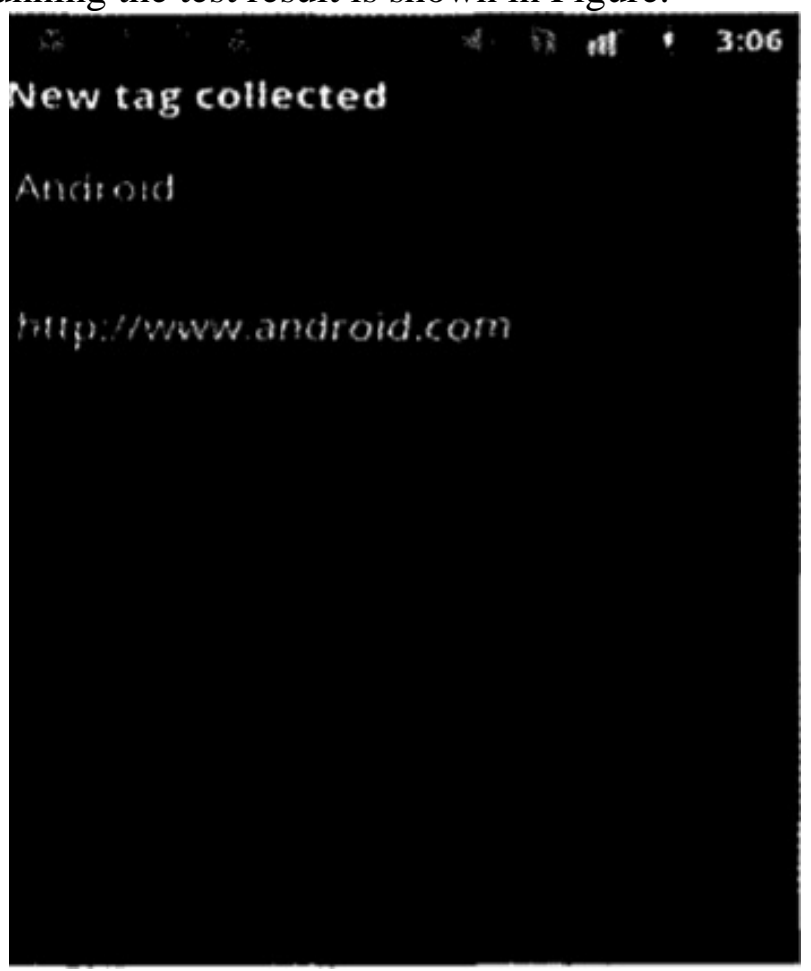

\section{Conclusion}

NFC is used in close range of smart devices quickly established among key technology in wireless communication. NFC not only can be used for identification, but also can be in two-way 
data interaction between two wireless smart devices. In addition to information exchange, NFC for mobile Internet devices provides a security mechanism that allows users to not be limited by time and space, and it can easily identify and transport a wide variety of data and information. Insofar as NFC device is effective distance range, the two sides will be smart devices automatically secure authentication and network communications. Users do not need to rely on specific applications and settings. This fast authentication and then through NFC Bluetooth , Wi-Fi and other high-speed, long-distance transmission of wireless devices for data transmission mode, enables fast, safe establish long-distance, high-speed data communication between devices, enabling advertising service information, location information data acquisition, contactless mobile payment and identification functions. NFC has a broad application prospect, especially in the combination and Android smartphone operating system, it will give full play to the function of the NFC.

\section{References}

[1]. Vedat Coskun, Kerem OK, Busra Ozdenizci. Et al. Professional NFC Application Development for Android. Wrox;1(2013.4.22).

[2]. Information on: http://www.nfc-forum.org/spec_list/.

[3]. Information on:

http://developer.android,com/reference/android/nfc/package-summary.html.

[4]. IOS/IEC 14443, Identification cards — Contactless integrated circurt cards — proximity cards[S]. 2008. 